\title{
Clinical potential of resistin as a novel prognostic biomarker for cellulitis
}

\author{
AYSE ERTURK $^{1}$, MEDINE CUMHUR CURE $^{2}$, ERKAN CURE $^{3}$, AYSEL KURT $^{4}$, \\ AYSEGUL COPUR CICEK ${ }^{5}$ and SULEYMAN YUCE ${ }^{3}$
}

\author{
Departments of ${ }^{1}$ Infectious Disease, ${ }^{2}$ Biochemistry, ${ }^{3}$ Internal Medicine, ${ }^{4}$ Thoracic Surgery and ${ }^{5}$ Microbiology, \\ School of Medicine, Recep Tayyip Erdogan University, Rize 53200, Turkey
}

Received August 6, 2014; Accepted February 11, 2015

DOI: $10.3892 / \mathrm{etm} .2015 .2311$

\begin{abstract}
Cellulitis is an acute, subacute or chronic inflammation of the dermis and subdermal tissues, which is typically caused by bacteria, although other causes are possible. The present study aimed to evaluate the association between resistin levels and the recovery time of patients with cellulitis. In addition, the effect of resistin and insulin resistance on the prognosis of cellulitis was investigated. In total, 52 patients with cellulitis (male, 21; female, 31) and an age-matched group of 42 healthy individuals (male, 18; female, 24) were included in the study. The levels of serum resistin, fasting plasma glucose (FPG), homeostasis model assessment-insulin resistance (HOMA-IR), C-reactive protein (CRP) and other biochemical parameters were compared between the groups. The mean resistin levels in the cellulitis and control groups were $9.4 \pm 5.3$ and $5.8 \pm 3.1 \mathrm{ng} / \mathrm{ml}$, respectively. The levels of resistin, FPG, HOMA-IR and CRP were significantly higher in the cellulitis group compared with the control group $(\mathrm{P}<0.001)$. Furthermore, the mean recovery time of the patients with cellulitis was $21.2 \pm 5.6$ days. Thus, increased levels of resistin $(\mathrm{P}=0.002)$ and HOMA-IR $(\mathrm{P}=0.005)$ could be used as predictive factors for the recovery time. The enhanced levels of resistin and HOMA-IR were shown to correlate with the high CRP levels in the cellulitis group. Therefore, the results indicated that increased levels of resistin may function as a prognostic marker for cellulitis.
\end{abstract}

\section{Introduction}

Cellulitis is classified as an acute, subacute or chronic inflammation of the dermis and subdermal tissues, which is generally caused by a bacterial infection. The clinical manifestations of cellulitis include erythema, swelling, local tenderness of

Correspondence to: Dr Ayse Erturk, Department of Infectious Disease, School of Medicine, Recep Tayyip Erdogan University, 74 İslampaşa Mahallesi, Rize 53200, Turkey

E-mail: ayseerturk25@gmail.com

Key words: resistin, insulin resistance, cellulitis, C-reactive protein the skin and subcutaneous tissues, in addition to fever and malaise (1). Group A Streptococcus and Staphylococcus aureus are responsible for the majority of cellulitis cases (2). The clinical severity of cellulitis ranges from a mild infection, treatable with oral antibiotics, to a severe necrotizing infection associated with a high mortality rate (3).

Resistin is an adipocytokine that is released by adipocytes, muscles cells, pancreatic islet cells, mononuclear cells, macrophages and neutrophils (4). Resistin competes with lipopolysaccharides through binding to Toll-like receptors and may function as a proinflammatory cytokine in human monocytes. In addition, resistin is a member of the cysteine-rich secretory protein family, members of which are also known as resistin-like or 'found in inflammatory zone' molecules (5). Serum resistin levels have been observed to increase during severe bacterial and viral infections (6-8). Furthermore, increased resistin levels have been associated with insulin resistance (9), and a strong correlation has been observed between C-reactive protein (CRP) and resistin (10).

Hepatocyte-derived CRP is a sensitive indicator of inflammatory and infectious processes in a variety of tissues. CRP is synthesized in the liver under the regulation of interleukin (IL)-6 (11), and serves a crucial role in inflammation and acute-phase reactions. High levels of CRP have been shown to correlate with insulin resistance in the absence of diabetes mellitus and severe inflammation (12). A CRP response is observed during the progression of cellulitis $(11,13)$. However, using the CRP response as a biomarker for cellulitis has a number of disadvantages, including a delayed release and low specificity. Thus, numerous biomarkers, including resistin, have been studied in association with infectious diseases $(8,14-16)$.

The aim of the present pilot study was to investigate whether levels of resistin and insulin are effective prognostic indicators for estimating the recovery time of patients with cellulitis.

\section{Materials and methods}

Study population. The current observational cross-sectional study was conducted in the Department of Infectious Diseases in the Medical Faculty at Recep Tayyip Erdogan University (Rize, Turkey). In total, 52 patients diagnosed with cellulitis 
(female, 31; male, 21) were recruited. In addition, a control group was included, consisting of 42 healthy individuals (female, 24; male, 18) with no known infectious or chronic diseases, such as diabetes, hypertension, hyperlipidemia, coronary artery disease, chronic obstructive pulmonary disease or chronic renal failure. None of the subjects included in the study consumed alcohol, smoked or took drugs. The study was conducted in accordance with the Helsinki Declaration, and was approved by the local Ethics Committee of Rcep Tayyip Erdogan University (Rize, Turkey). Informed consent was obtained from all the participants.

Diagnosis of cellulitis. Cellulitis was diagnosed by an infectious disease specialist, according to the following criteria: i) Edema, rash, tenderness and hotness of the skin in the lower extremities; ii) elevated white blood cell (WBC) count, erythrocyte sedimentation rate (ESR) and CRP levels (however, normal values of these tests did not automatically exclude the diagnosis of cellulitis); iii) cultivation of the infectious agent from an aspirate or biopsy material; and iv) exclusion of the presence of deep vein thrombosis (DVT) using superficial ultrasonography (USG) and Doppler USG $(1,17)$.

Diagnosis of DVT for differential diagnosis. DVT was defined by a radiologist as a reduction in the calibration of the femoral and popliteal veins. Furthermore, DVT was visualized as an intraluminal isoechoic thrombus, possessing a reflux with poor recanalization, which is a typical symptom of DVT (18). These examinations were performed using Doppler USG with a $10 \mathrm{MHz}$ direct ultrasound probe (Xario; Toshiba Medical Systems Corporation, Tokyo, Japan).

Cellulitis recovery period. Recovery from cellulitis was determined according to previously defined guidelines $(19,20)$. Briefly, recovery was classified as an improvement in clinical symptoms and the complete recovery from edema, rash, tenderness and hotness of the skin and soft tissues. The recovery time was estimated in accordance with the previously described guidelines.

Laboratory measurements. Biochemical parameters of the subjects were measured following a 12-h fast. Serum samples were stored at $-30^{\circ} \mathrm{C}$. The levels of serum fasting plasma glucose (FPG), blood urea nitrogen, creatinine, aspartate aminotransferase, alanine aminotransferase and other biochemical parameters were determined using photometric and spectrophotometric assays using the Architect c16000 chemical analyzer (Abbott Diagnostics, Chicago, IL, USA). CRP levels were measured via the nephelometric method using an IMMAGE 800 system (Beckman Coulter, Inc., Brea, CA, USA). Hematological measurements, such as the levels of WBCs, platelets and hemoglobin, were acquired using a CELL-DYN Ruby hematology system (Abbott Diagnostics). Insulin levels were measured using a chemiluminescent microparticle immunoassay system (Architect Immunoassay Analyzer; Abbott Diagnostics).

Resistin level measurement. Serum resistin levels were quantified using a commercially available enzyme-linked immunosorbent assay (ELISA) kit (RayBiotech, Inc., Norcross,
GA, USA), according to the manufacturer's instructions. Absorbance was measured at a wavelength of $450 \mathrm{~nm}$, using an ELISA reader (Multiskan ${ }^{\mathrm{TM}}$ Go, Thermo Fisher Scientific, Vantaa, Finland). Resistin levels are presented in units of $\mathrm{ng} / \mathrm{ml}$. The intra-assay and inter-assay coefficients of variation were $<10$ and $<12 \%$, respectively, and the sensitivity was calculated as $2 \mathrm{pg} / \mathrm{ml}$.

Homeostasis model assessment-insulin resistance (HOMA-IR) score. The HOMA-IR score was calculated using the following formula (21): HOMA-IR = [FPG $(\mathrm{mmol} / \mathrm{l}) \mathrm{x}$ fasting serum insulin $(\mathrm{mU} / \mathrm{ml})] / 22.5$. The cut-off value of the HOMA-IR score was 2.7 .

Statistical analysis. Results are presented as the mean \pm standard deviation. Statistical analyses were performed using SPSS statistical software for Windows, version 13.0 (SPSS, Inc., Chicago, IL, USA). The Kolmogorov-Smirnov test was applied to all the data to determine the normal and non-normal data distributions. Foreach parameter, the statistical significance of the difference between the patient and control groups was analyzed using the independent-samples t-test for normally distributed parameters and the Mann-Whitney U test for non-normally distributed parameters. Subgroup analyses for gender were performed using nested analysis of variance, followed by Bonferroni analysis. The associations between variables were analyzed using Pearson's correlation analysis. Multivariate (MVA) logistic regression analyses were performed to determine the independent associations between the various subject parameters and the cellulitis recovery time. The results are expressed as odds ratios (OR) with a $95 \%$ confidence interval $(\mathrm{CI}) . \mathrm{P}<0.05$ was considered to indicate a statistically significant difference.

\section{Results}

Subject characteristics and laboratory measurements. The mean age of the patients in the cellulitis and control groups was $61.3 \pm 13.7$ and $61.5 \pm 7.7$ years, respectively. The mean recovery time for patients with cellulitis was $21.1 \pm 5.6$ days (range, 12-39 days). The parameter measurements for the cellulitis group were as follows: Body mass index (BMI), $28.9 \pm 3.9 \mathrm{~kg} / \mathrm{m}^{2}$; resistin level, $9.4 \pm 5.3 \mathrm{ng} / \mathrm{ml}$; FPG level, $100.6 \pm 19.3 \mathrm{mg} / \mathrm{dl}$; insulin level, $10.9 \pm 2.2 \mu \mathrm{IU} / \mathrm{ml}$; HOMA-IR, 2.6 \pm 0.7 ; ESR, $37.1 \pm 25.2 \mathrm{~mm} / \mathrm{h}$; CRP level, $9.3 \pm 8.3 \mathrm{mg} / \mathrm{dl}$; and $\mathrm{WBC}$ count, $11.7 \pm 3.3 \times 10^{9} / 1$. The measurements for the control group were as follows: BMI, $28.6 \pm 4.8 \mathrm{~kg} / \mathrm{m}^{2}$; resistin level, 5.8 $\pm 3.1 \mathrm{ng} / \mathrm{ml}$; FPG level, $89.6 \pm 8.2 \mathrm{mg} / \mathrm{dl}$; insulin

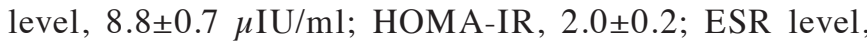
$16.5 \pm 14.4 \mathrm{~mm} / \mathrm{h}$; CRP level, $0.7 \pm 0.6 \mathrm{mg} / \mathrm{dl}$; and WBC count, $7.3 \pm 2.5 \times 10^{9} / 1$. The levels of resistin, FPG, insulin and CRP, the HOMA-IR, the ESR and the WBC count were significantly higher in the cellulitis group when compared with the control group $(\mathrm{P}<0.001)$. The demographic characteristics and biochemical parameters of the subjects are presented in Table I.

Correlation analysis. Pearson's correlation analyses indicated positive correlations when comparing the resistin level with the BMI $(\mathrm{r}=0.398, \mathrm{P}=0.003)$, the FPG level $(\mathrm{r}=0.345, \mathrm{P}=0.002)$, 
Table I. Characteristics and laboratory parameters for the two groups.

\begin{tabular}{|c|c|c|c|}
\hline Parameter & Cellulitis $(n=52)$ & Control $(n=42)$ & P-value \\
\hline Age (years) & $61.3 \pm 13.7$ & $61.5 \pm 7.7$ & 0.919 \\
\hline Gender, M/F (n) & $21 / 31$ & $18 / 24$ & 0.403 \\
\hline $\operatorname{BMI}\left(\mathrm{kg} / \mathrm{m}^{2}\right)$ & $28.9 \pm 3.9$ & $28.6 \pm 4.8$ & 0.645 \\
\hline Resistin (ng/ml) & $9.4 \pm 5.3$ & $5.8 \pm 3.1$ & 0.001 \\
\hline FPG (mg/dl) & $100.6 \pm 19.3$ & $89.6 \pm 8.2$ & 0.001 \\
\hline Insulin $(\mu \mathrm{IU} / \mathrm{ml})$ & $10.9 \pm 2.2$ & $8.8 \pm 0.7$ & 0.001 \\
\hline HOMA-IR & $2.6 \pm 0.7$ & $2.0 \pm 0.2$ & 0.001 \\
\hline WBC (x109/1) & $11.7 \pm 3.3$ & $7.3 \pm 2.5$ & 0.001 \\
\hline ESR (mm/h) & $37.1 \pm 25.2$ & $16.5 \pm 14.4$ & 0.001 \\
\hline CRP (mg/dl) & $9.3 \pm 8.3$ & $0.7 \pm 0.6$ & 0.001 \\
\hline BUN (mg/dl) & $37.6 \pm 18.3$ & $36.4 \pm 10.8$ & 0.718 \\
\hline Creatinin (mg/dl) & $0.8 \pm 0.3$ & $0.8 \pm 0.2$ & 0.773 \\
\hline AST (IU/l) & $26.1 \pm 15.5$ & $23.2 \pm 9.6$ & 0.314 \\
\hline ALT (IU/1) & $26.5 \pm 19.3$ & $22.1 \pm 15.6$ & 0.280 \\
\hline TC (mg/dl) & $223.4 \pm 52.0$ & $189.6 \pm 32.2$ & 0.001 \\
\hline TG (mg/dl) & $131.4 \pm 47.2$ & $120.4 \pm 57.0$ & 0.401 \\
\hline HDL (mg/dl) & $46.1 \pm 15.7$ & $45.9 \pm 14.7$ & 0.966 \\
\hline LDL (mg/dl) & $129.8 \pm 36.4$ & $119.0 \pm 27.5$ & 0.149 \\
\hline
\end{tabular}

Data are expressed as the mean \pm standard deviation. M, male; F, female; BMI, body mass index; FPG, fasting plasma glucose; HOMA-IR, homeostasis model assessment-insulin resistance; WBC, white blood cell; ESR, erythrocyte sedimentation rate; CRP, C-reactive protein; BUN, blood urea nitrogen; AST, aspartate aminotransferase; ALT, alanine aminotransferase; TC, total cholesterol; TG, triglyceride; HDL, high-density lipoprotein; LDL low-density lipoprotein.

Table II. Pearson correlation coefficients (r) between resistin levels, recovery time and measured parameters in patients with cellulitis.

\begin{tabular}{lccccc}
\hline & \multicolumn{2}{c}{ Resistin levels } & & \multicolumn{2}{c}{ Recovery time } \\
\cline { 2 - 3 } \cline { 5 - 6 } Parameters & R-value & P-value & & R-value & P-value \\
\hline Resistin & - & - & & 0.547 & 0.001 \\
BMI & 0.398 & 0.003 & & 0.106 & 0.097 \\
FPG & 0.345 & 0.002 & & 0.336 & 0.003 \\
Insulin & 0.541 & 0.001 & & 0.500 & 0.001 \\
HOMA-IR & 0.622 & 0.001 & & 0.575 & 0.001 \\
CRP & 0.438 & 0.001 & & 0.531 & 0.001 \\
TC & 0.275 & 0.015 & & 0.360 & 0.001 \\
\hline
\end{tabular}

BMI, body mass index; FPG, fasting plasma glucose; HOMA-IR, homeostasis model assessment-insulin resistance; CRP, C-reactive protein; TC, total cholesterol.

the insulin level $(\mathrm{r}=0.541, \mathrm{P}<0.001)$, the HOMA-IR score $(\mathrm{r}=0.622, \mathrm{P}<0.001)$, the CRP level $(\mathrm{r}=0.438, \mathrm{P}<0.001)$ and the total cholesterol level $(\mathrm{r}=0.275, \mathrm{P}=0.015)$. Furthermore, positive correlations were observed between the patient recovery time and the levels of resistin $(r=0.547, \mathrm{P}<0.001), \mathrm{CRP}$ $(\mathrm{r}=0.531, \mathrm{P}<0.001)$, FPG $(\mathrm{r}=0.336, \mathrm{P}=0.003)$ and HOMA-IR $(\mathrm{r}=0.575, \mathrm{P}<0.001$; Table II).
Table III. Multivariate logistic regression analysis of the independent variables associated with the recovery time in patients with cellulitis.

\begin{tabular}{lccc}
\hline Parameter & OR & $95 \%$ CI & P-value \\
\hline Resistin & 0.649 & $0.213-0.967$ & 0.002 \\
CRP & 0.396 & $0.101-0.512$ & 0.001 \\
ESR & 0.140 & $0.053-0.227$ & 0.002 \\
WBC & 1.048 & $0.582-1.514$ & 0.001 \\
HOMA-IR & 0.343 & $0.425-2.262$ & 0.005 \\
BMI & 0.450 & $0.335-0.728$ & 0.002 \\
Age & 0.513 & $0.214-0.719$ & 0.992 \\
Gender & 1.343 & $0.784-2.600$ & 0.498 \\
Hb & 0.326 & $0.288-0.461$ & 0.501 \\
Platelet & 0.323 & $0.178-0.359$ & 0.746 \\
Glucose & 0.190 & $0.135-0.218$ & 0.904 \\
AST & 0.082 & $0.030-0.977$ & 0.337 \\
ALT & 0.040 & $0.009-0.132$ & 0.949 \\
BUN & 0.029 & $0.004-0.136$ & 0.724 \\
Creatinine & 0.650 & $0.150-1.99$ & 0.131 \\
\hline
\end{tabular}

CRP, C-reactive protein; ESR, erythrocyte sedimentation rate; WBC, white blood cell; HOMA-IR, homeostasis model assessment-insulin resistance; BMI, body mass index; $\mathrm{Hb}$, hemoglobin; AST, aspartate aminotransferase; ALT, alanine aminotransferase; BUN, blood urea nitrogen; OR, odds ratio; 95\% CI, 95\% confidence interval. 
Table IV. Subgroup analysis of the gender groups by analysis of variance.

\begin{tabular}{lcccc}
\hline & \multicolumn{2}{c}{ Cellulitis group } & \multicolumn{2}{c}{ Control group } \\
\cline { 2 - 3 } Parameter & Male & Female & Male & Female \\
\hline Resistin (ng/ml) & $7.8 \pm 3.7^{\mathrm{a}}$ & $10.5 \pm 5.9$ & $5.0 \pm 2.4^{\mathrm{b}}$ & $6.6 \pm 3.4^{\mathrm{c}}$ \\
FPG (mg/dl) & $99.2 \pm 19.1$ & $101.5 \pm 19.7$ & $89.0 \pm 8.5^{\mathrm{d}}$ & $90.2 \pm 8.2^{\mathrm{e}}$ \\
HOMA-IR & $2.4 \pm 0.6$ & $2.8 \pm 0.7^{\mathrm{f}}$ & $1.9 \pm 0.2^{\mathrm{b}, \mathrm{g}}$ & $2.0 \pm 0.2^{\mathrm{b}, \mathrm{h}}$ \\
CRP (mg/dl) & $6.2 \pm 4.9$ & $11.4 \pm 9.4^{\mathrm{i}}$ & $0.7 \pm 0.9^{\mathrm{b}, \mathrm{j}}$ & $0.6 \pm 0.4^{\mathrm{b}, \mathrm{k}}$ \\
WBC (x 10 $/ \mathrm{l})$ & $12.0 \pm 3.3$ & $11.5 \pm 3.3$ & $8.6 \pm 3.0^{1, \mathrm{~m}}$ & $6.1 \pm 1.1^{\mathrm{b}, \mathrm{n}, \mathrm{o}}$ \\
\hline
\end{tabular}

${ }^{\mathrm{a}} \mathrm{P}=0.041,{ }^{\mathrm{b}} \mathrm{P}<0.001,{ }^{\mathrm{c}} \mathrm{P}=0.009,{ }^{\mathrm{d}} \mathrm{P}=0.031$ and ${ }^{\mathrm{e}} \mathrm{P}=0.039$ vs. female cellulitis group; ${ }^{\mathrm{f}} \mathrm{P}=0.011$ vs. male cellulitis group; ${ }^{\mathrm{g}} \mathrm{P}=0.028,{ }^{\mathrm{h}} \mathrm{P}=0.036$, ${ }^{i} \mathrm{P}=0.006,{ }^{\mathrm{j}} \mathrm{P}=0.025,{ }^{\mathrm{k}} \mathrm{P}=0.017$ and ${ }^{\mathrm{l}} \mathrm{P}=0.003$, vs. male cellulitis group; ${ }^{\mathrm{m}} \mathrm{P}=0.007$ vs. female cellulitis group; ${ }^{\mathrm{n}} \mathrm{P}<0.001$ vs. male cellulitis group; ${ }^{\circ} \mathrm{P}=0.039$, vs. male control group. FPG, fasting plasma glucose; HOMA-IR, homeostasis model assessment-insulin resistance; CRP, C-reactive protein; WBC, white blood cell.

MVA analysis. MVA analyses were performed with the recovery time as the dependent variable and the resistin level, FPG level, HOMA-IR, CRP level, ESR, WBC count, age, BMI and gender as independent variables. The following parameters were observed to be independently associated with the recovery time: Resistin level (OR, 0.649; 95\% CI, 0.213-0.967; $\mathrm{P}=0.002$ ), CRP (OR, 0.396; 95\% CI, 0.101-0.512; $\mathrm{P}=0.001$ ), WBC count (OR, 1.048; 95\% CI, 0.582-1.514; $\mathrm{P}<0.001)$, BMI (OR, 0.450; 95\% CI, 0.335-0.728; $\mathrm{P}=0.002$ ) and HOMA-IR score (OR, 0.343; 95\% CI, 0.425-2.262; $\mathrm{P}=0.005)$. The $\mathrm{P}$-values of the remaining parameters were not significant. All the results are presented in Table III.

Subgroup analyses. The study population was divided according to gender into cellulitis male (CEM), control male $(\mathrm{COM})$, cellulitis female (CEF) and control female (COF) subgroups. The resistin levels in the CEF group were significantly higher when compared with the CEM $(\mathrm{P}=0.041)$, COM $(\mathrm{P}<0.001)$ and $\mathrm{COF}(\mathrm{P}=0.009)$ groups. The HOMA-IR score in the CEM group was significantly higher compared with the COM $(\mathrm{P}=0.028)$ and $\mathrm{COF}(\mathrm{P}=0.036)$ groups. In addition, HOMA-IR in the CEF group was significantly higher compared with the $\mathrm{COM}(\mathrm{P}<0.001)$ and $\mathrm{COF}(\mathrm{P}<0.001)$ groups (Table IV).

\section{Discussion}

In the present study, the levels of resistin, FPG, insulin and CRP, as well as the HOMA-IR score, ESR and WBC count were significantly higher in the patients with cellulitis compared with the healthy control subjects. MVA regression analysis indicated marked independent associations between the cellulitis recovery time and high levels of serum resistin, HOMA-IR, CRP and WBCs. Similarly, resistin levels were shown to positively correlate with the serum FPG, insulin, HOMA-IR and CRP levels. Thus, the results of the present study indicate that the HOMA-IR score and resistin level correlate markedly with the CRP level and the recovery time of patients with cellulitis. High levels of these parameters were observed to be concomitant with a long recovery time. Thus, resistin level may be an effective prognostic marker for estimating the recovery time of patients with cellulitis. A previous study reported an association between enhanced levels of CRP and those of resistin in patients with inflammatory diseases, and hypothesized that these increases may indicate disease activity (22). The present study also demonstrated a strong correlation between the levels of CRP and resistin. CRP is a useful marker of the severity of an infection, and resistin may function similarly as a prognostic factor.

Previous studies have reported that resistin may indicate the severity of the disease in patients with neonatal sepsis, in a similar manner to IL-6 and CRP $(7,14,23,24)$. Furthermore, recent studies have demonstrated that resistin may serve a function in inflammation and autoimmunity $(5,25)$. Bokarewa et al reported that the use of recombinant resistin resulted in a marked upregulation in the genes for tumor necrosis factor (TNF)- $\alpha$ and IL-6 (26). A number of studies have indicated a marked correlation between the levels of resistin and CRP in severe inflammatory diseases $(22,27,28)$. From the results of the present study, it can be hypothesized that resistin is released by macrophages and adipose tissue during cellulitis infection, after which the levels of resistin are further increased by inflammatory processes. Furthermore, increased inflammation and the consequent elevation in resistin levels may result in the development of insulin resistance.

Notably, subgroup analyses revealed the CRP response in females with cellulitis to be higher compared with that in male patients. Furthermore, resistin and insulin resistance levels in the female cellulitis patients were increased compared with the male patients. Previous studies have reported the resistin levels of healthy female individuals to be higher compared with those in healthy males $(29,30)$. Thus, resistin may be more useful as a prognostic factor in female patients with cellulitis. By contrast, in an alternative study, high resistin levels were reported in male and female patients with chronic obstructive lung disease (31). In addition, the results of present study indicated an increase in the levels of resistin in male patients with cellulitis. Thus, resistin may function as a prognostic factor in males with cellulitis, in addition to females. However, the limited population sample size in the present study may have affected the results. Further studies including a broader 
study population are required to elucidate the difference in the resistin response between male and female patients with cellulitis.

During cellulitis infection, the skin accumulates populations of lymphoid and reticular cells with the capacity to secrete lymphocytes and cytokines. These secretions rapidly reduce the number of viable bacteria by enhancing the ability of circulating macrophages and neutrophils to infiltrate the skin (32). Furthermore, during a cellulitis infection, M1 macrophages infiltrate the adipose tissue and subsequently secrete proinflammatory cytokines and generate reactive oxygen species, which in turn recruit more macrophages and amplify the inflammatory response $(33,34)$. Thus, resistin and cytokines may be released from the adipose tissue, causing an increase in the levels of CRP. In the present study, the levels of CRP and resistin were enhanced in the patients with cellulitis compared with the healthy subjects, which may have been in response to inflammation.

Hyperglycemia has been associated with increased levels of cytokines and counter-regulatory hormones, which may result in the development of insulin resistance. A previous study revealed an association among resistin levels, insulin resistance and obesity (34). TNF- $\alpha$ and IL-6 have been shown to alter the expression of peroxisome proliferator-activated receptor (PPAR) $-\gamma$ in adipocytes $(35,36)$. PPAR $-\gamma$ is an anti-inflammatory nuclear protein with insulin-sensitizing functions. CRP enhances the inflammatory response, and causes systemic inflammation (37). Plasma CRP levels have been shown to correlate with circulating levels of other inflammatory biomarkers (38). Furthermore, a strong positive correlation has been observed between plasma CRP levels and insulin resistance (39). Previous studies have reported that plasma resistin levels correlate positively with insulin resistance, obesity and glucose intolerance $(40,41)$. The proinflammatory effects of resistin were attributed to its ability to activate the nuclear factor- $\kappa \mathrm{B}$ signaling pathway and subsequently enhance the production of proinflammatory cytokines, including TNF- $\alpha$ and IL-6, which impair insulin-signaling pathways and result in the development of insulin resistance $(42,43)$. In addition, increased levels of proinflammatory cytokines and CRP may lead to insulin resistance. In the current study, CRP levels were higher in patients with cellulitis compared with the healthy control subjects. Thus, increased levels of CRP may also be associated with higher proinflammatory cytokine levels. A higher HOMA-IR score may mediate the effects of resistin and proinflammatory cytokines.

There were a number of limitations to the present study. As a pilot study, the sample size was small and did not represent the general population. In addition, serum samples were obtained from the patients with active cellulitis. It may have been useful to obtain samples from patients following recovery from cellulitis, in order to compare the resistin and CRP levels with the healthy levels. Thus, further investigations are required.

In conclusion, the levels of resistin and the HOMA-IR score were higher in the cellulitis group when compared with the control group. In addition, positive correlations between the resistin level and HOMA-IR with the CRP level were observed in the patients with cellulitis. Serum resistin levels may increase during cellulitis infection due to enhanced levels of proinflammatory cytokines. Consequently, insulin resistance and hyperinsulinemia may develop in patients with cellulitis. The results of the present study indicate that increased resistin levels may provide a novel prognostic factor for cellulitis.

\section{References}

1. Bailey E and Kroshinsky D: Cellulitis: diagnosis and management. Dermatol Ther 24: 229-239, 2011.

2. Muileboom J, Hamilton M and Kelly L: The changing face of cellulitis and MRSA in rural Canada: a clinical update. Can J Rural Med 18: 137-139, 2013.

3. Stevens DL, Bisno AL, Chambers HF, Dellinger EP, Goldstein EJC, Gorbach SL, Hirschmann JV, Kaplan SL, Montoya JG and Wade JC: Practice guidelines for the diagnosis and management of skin and soft tissue infections: 2014 update by the Infectious Diseases Society of America. Clin Infect Dis 59: e10-e52, 2014

4. Szalowska E, Elferink MGL, Hoek A, Groothuis GMM and Vonk RJ: Resistin is more abundant in liver than adipose tissue and is not up-regulated by lipopolysaccharide. J Clin Endocrinol Metab 94: 3051-3057, 2009.

5. Gan AM, Butoi ED, Manea A, Simion V, Stan D, Parvulescu MM, Calin M, Manduteanu I and Simionescu M: Inflammatory effects of resistin on human smooth muscle cells: up-regulation of fractalkine and its receptor, CX3CR 1 expression by TLR4 and Gi-protein pathways. Cell Tissue Res 351: 161-174, 2013.

6. Durazzo M, Belci P, Niro G, et al: Variations of serum levels of adiponectin and resistin in chronic viral hepatitis. J Endocrinol Invest 36: 600-605, 2013.

7. Vassiliadi DA, Tzanela M, Kotanidou A, Orfanos SE, Nikitas N, Armaganidis A, Koutsilieris M, Roussos C, Tsagarakis S and Dimopoulou I: Serial changes in adiponectin and resistin in critically ill patients with sepsis: associations with sepsis phase, severity, and circulating cytokine levels. J Crit Care 27: 400-409, 2012.

8. Cekmez F, Canpolat FE, Cetinkaya M, Aydinöz S, Aydemir G, Karademir F, Ipcioglu OM and Sarici SÜ: Diagnostic value of resistin and visfatin, in comparison with C-reactive protein, procalcitonin and interleukin-6 in neonatal sepsis. Eur Cytokine Netw 22: 113-117, 2011.

9. Gerber PA, Thalhammer C, Schmied C, Spring S, Amann-Vesti B, Spinas GA and Berneis K: Small, dense LDL particles predict changes in intima media thickness and insulin resistance in men with type 2 diabetes and prediabetes - a prospective cohort study. PLoS One 8: e72763, 2013.

10. Makni E, Moalla W, Benezzeddine-Boussaidi L, Lac G, Tabka Z and Elloumi M: Correlation of resistin with inflammatory and cardiometabolic markers in obese adolescents with and without metabolic syndrome. Obes Facts 6: 393-404, 2013.

11. Puel A, Picard C, Lorrot M, Pons C, Chrabieh M, Lorenzo L, Mamani-Matsuda M, Jouanguy E, Gendrel D and Casanova JL: Recurrent staphylococcal cellulitis and subcutaneous abscesses in a child with autoantibodies against IL-6. J Immunol 180: 647-654, 2008.

12. Gruzdeva O, Uchasova E, Dyleva Y, Belik E, Shurygina E and Barbarash O: Insulin resistance and inflammation markers in myocardial infarction. J Inflamm Res 6: 83-90, 2013.

13. Karppelin M, Siljander T, Aittoniemi J, Hurme M, Huttunen R, Huhtala H, KereJ, Vuopio J and Syrjänen J: Predictors of recurrent cellulitis in five years. Clinical risk factors and the role of PTX3 and CRP. J Infect: Nov 15, 2014 (Epub ahead of print).

14. Kitanovski L, Jazbec J, Hojker S and Derganc M: Diagnostic accuracy of lipopolysaccharide-binding protein for predicting bacteremia/clinical sepsis in children with febrile neutropenia: comparison with interleukin-6, procalcitonin, and C-reactive protein. Support Care Cancer 22: 269-277, 2014.

15. Park HK, Qatanani M, Briggs ER, Ahima RS and Lazar MA: Inflammatory induction of human resistin causes insulin resistance in endotoxemic mice. Diabetes 60: 775-783, 2011.

16. Sakr Y, Sponholz C, Tuche F, Brunkhorst F and Reinhart K: The role of procalcitonin in febrile neutropenic patients: review of the literature. Infection 36: 396-407, 2008.

17. Hirschmann JV and Raugi GJ: Lower limb cellulitis and its mimics: part I. Lower limb cellulitis. J Am Acad Dermatol 67: e1-e12, 2012. 
18. Maze MJ, Skea S, Pithie A, Metcalf S, Pearson JF and Chambers ST: Prevalence of concurrent deep vein thrombosis in patients with lower limb cellulitis: a prospective cohort study. BMC Infect Dis 13: 141, 2013.

19. Stevens DL, Bisno AL, Chambers HF, et al; Infectious Diseases Society of America: Practice guidelines for the diagnosis and management of skin and soft-tissue infections. Clin Infect Dis 41: 1373-1406, 2005.

20. May AK, Stafford RE, Bulger EM, Heffernan D, Guillamondegui O, Bochicchio G and Eachempati SR; Surgical Infection Society: Treatment of complicated skin and soft tissue infections. Surg Infect (Larchmt) 10: 467-499, 2009.

21. Matthews DR, Hosker JP, Rudenski AS, Naylor BA, Treacher DF and Turner RC: Homeostasis model assessment: insulin resistance and beta-cell function from fasting plasma glucose and insulin concentrations in man. Diabetologia 28: 412-419, 1985.

22. Fadda SM, Gamal SM, Elsaid NY and Mohy AM: Resistin in inflammatory and degenerative rheumatologic diseases. Relationship between resistin and rheumatoid arthritis disease progression. Z Rheumatol 72: 594-600, 2013.

23. Aliefendioglu D, Gürsoy T, Çağlayan O, Aktaş A and Ovalı F: Can resistin be a new indicator of neonatal sepsis? Pediatr Neonatol 55: 53-57, 2014.

24. Gokmen Z, Ozkiraz S, Kulaksizoglu S, Kilicdag H, Ozel D, Ecevit A and Tarcan A: Resistin - a novel feature in the diagnosis of sepsis in premature neonates. Am J Perinatol 30: 513-517, 2013

25. Tanaka N, Kusunoki N, Kusunoki Y, Hasunuma T and Kawai S: Resistin is associated with the inflammation process in patients with systemic autoimmune diseases undergoing glucocorticoid therapy: comparison with leptin and adiponectin. Mod Rheumatol 23: 8-18, 2013.

26. PubMed PMID: 22434063. Bokarewa M, Nagaev I, Dahlberg L, Smith U and Tarkowski A: Resistin, an adipokine with potent proinflammatory properties. J Immunol 174: 5789-5795, 2005.

27. de Luis DA, González Sagrado M, Conde R, Aller R, Izaola O and Castro MJ: Circulating adipocytokines in morbid obese patients, relation with cardiovascular risk factors and anthropometric parameters. Nutr Hosp 26: 91-96, 2011.

28. Stofkova A: Resistin and visfatin: regulators of insulin sensitivity, inflammation and immunity. Endocr Regul 44: 25-36, 2010.

29. Yoshino T, Kusunoki N, Tanaka N, Kaneko K, Kusunoki Y, Endo H, Hasunuma T and Kawai S: Elevated serum levels of resistin, leptin, and adiponectin are associated with C-reactive protein and also other clinical conditions in rheumatoid arthritis. Intern Med 50: 269-275, 2011.

30. Chen BH, Song Y, Ding EL, Roberts CK, Manson JE, Rifai N, Buring JE, Gaziano JM and Liu S: Circulating levels of resistin and risk of type 2 diabetes in men and women: results from two prospective cohorts. Diabetes Care 32: 329-334, 2009.
31. Breyer MK, Rutten EP, Vernooy JH, Spruit MA, Dentener MA, van der Kallen C, vanGreevenbroek MM and Wouters EF: Gender differences in the adipose secretome system in chronic obstructive pulmonary disease (COPD): a pivotal role of leptin. Respir Med 105: 1046-1053, 2011.

32. Sachs MK: Cutaneous cellulitis. Arch Dermatol 127: 493-496, 1991.

33. Rull A, Camps J, Alonso-Villaverde C and Joven J: Insulin resistance, inflammation, and obesity: role of monocyte chemoattractant protein-1 (or CCL2) in the regulation of metabolism. Mediators Inflamm 2010: 326580, 2010.

34. Goktas Z, Moustaid-Moussa N, Shen CL, Boylan M, Mo H and Wang S: Effects of bariatric surgery on adipokine-induced inflammation and insulin resistance. Front Endocrinol (Lausanne) 4: 69, 2013.

35. Fernández-Veledo S, Nieto-Vazquez I, Vila-Bedmar R, Garcia-Guerra L,Alonso-Chamorro MandLorenzo M: Molecular mechanisms involved in obesity-associated insulin resistance: therapeutical approach. Arch Physiol Biochem 115: 227-239, 2009.

36. Zhou M, Wu R, Dong W, Jacob A and Wang P: Endotoxin downregulates peroxisome proliferator-activated receptor-gamma via the increase in TNF-alpha release. Am J Physiol Regul Integr Comp Physiol 294: R84-R92, 2008.

37. Wang $Z$ and Nakayama T: Inflammation, a link between obesity and cardiovascular disease. Mediators Inflamm 2010: 535918, 2010.

38. Kones R: Rosuvastatin, inflammation, C-reactive protein, JUPITER, and primary prevention of cardiovascular disease - a perspective. Drug Des Devel Ther 4: 383-413, 2010.

39. Pfützner A and Forst T: High-sensitivity C-reactive protein as cardiovascular risk marker in patients with diabetes mellitus. Diabetes Technol Ther 8: 28-36, 2006.

40. Choi SH, Hong ES and Lim S: Clinical implications of adipocytokines and newly emerging metabolic factors with relation to insulin resistance and cardiovascular health. Front Endocrinol (Lausanne) 4: 97, 2013.

41. Jankiewicz-Wika J, Kołomecki K, Cywiński J, Piestrzeniewicz K, Swiętosławski J, Stępień H and Komorowski J: Impact of vertical banded gastroplasty on body weight, insulin resistance, adipocytokine, inflammation and metabolic syndrome markers in morbidly obese patients. Endokrynol Pol 62: 109-119, 2011.

42. Zeyda M and Stulnig TM: Obesity, inflammation, and insulin resistance - a mini-review. Gerontology 55: 379-386, 2009.

43. Kumar R, Prakash S, Chhabra S, Singla V, Madan K, Gupta SD, Panda SK, Khanal S and Acharya SK: Association of pro-inflammatory cytokines, adipokines \& oxidative stress with insulin resistance \& non-alcoholic fatty liver disease. Indian J Med Res 136: 229-236, 2012. 